\title{
Detection of Inter-hemispheric Asymmetries of Brain Perfusion in SPECT
}

\author{
Bérengère Aubert-Broche ${ }^{1}$, Christophe Grova ${ }^{1},{\text { Pierre } \text { Jannin }^{1} \text {, Irène Buvat }}^{2}$, \\ Habib Benali ${ }^{2}$, and Bernard Gibaud ${ }^{1}$ \\ 1 Laboratoire IDM, Faculté de Médecine, Université de Rennes 1, France \\ \{berengere.broche, christophe.grova,pierre.jannin, bernard.gibaud\} \\ Quniv-rennes1.fr, \\ 2 INSERM U494, CHU Pitié Salpétrière, Paris \\ \{irene. buvat, habib.benali\}@imed.jussieu.fr
}

\begin{abstract}
This paper describes an unsupervised method to help detection of significant functional inter-hemispheric asymmetries in brain SPECT. A validation of this method was performed with realistic simulated SPECT data sets with known asymmetries (in size and amplitude) as gold standard. Detection performances were assessed through Receiver Operating Characteristic (ROC) analysis based on measures of the overlap between known and detected asymmetries.
\end{abstract}

\section{Introduction}

${ }^{99 m}$ Technetium HMPAO and ${ }^{99 m}$ Tc ECD Single Photon Emission Computed Tomography (SPECT) imaging is now commonly used to highlight altered regional cerebral perfusion. In partial epilepsy, SPECT imaging is considered relevant to show perfusion abnormalities, such as hyperperfusion in ictal SPECT (i.e. during epileptic seizure) and hypoperfusion in interictal SPECT (i.e. at a distance from any epileptic seizure) to help localize the epileptogenic focus [1].

Although in clinical routine the analysis of brain SPECT images is often limited to a qualitative side-by-side visual inspection of the data, recent research works have attempted to propose quantitative approaches. The relationship between blood flow and HMPAO or ECD SPECT brain uptake is non linear due to a saturation phenomenon. Absolute measurement of regional cerebral blood flow (rCBF) from HMPAO/ECD SPECT scans is thus not feasible. Only relative quantification can be used, for example using regions of interest (ROIs). ROIs may be geometric [2] or may follow the boundaries of anatomical structures [3]. They may be positioned manually or with the assistance of an anatomical atlas or template [4] or of the Magnetic Resonance images (MR) of the patient [5].

In this paper we propose a fully automatic method to detect inter-hemispheric asymmetries of brain perfusion in SPECT. The principle of this method is described as well as an assessment of its detection capabilities using realistic simulated SPECT data. 


\section{Material and Methods}

The proposed method aims at detecting inter-hemispheric functional asymmetries in brain SPECT images, based on anatomical information available from MR images. For this purpose, an asymmetry map was computed at the MRI spatial resolution. For each MRI voxel, the anatomically homologous voxel in the contro-lateral hemisphere was identified. Both homologous voxel coordinates were then mapped into the SPECT volume thanks to the SPECT-MRI registration. Neighborhoods were then defined around these SPECT voxels and compared to obtain a volume of inter-hemispheric differences from which we calculated a volume reflecting the significance of this difference (called "statistical volume").

The following sections describe the major steps of the method, i.e. the SPECTMRI registration, the identification of homologous voxels and the creation of the difference and statistical volumes. Then, we present the validation method and results based on realistic simulated SPECT data.

\subsection{Detection of Inter-hemispheric Functional Asymmetries}

SPECT-MRI registration: Rigid registration was performed using mutual information as described by Maes et al. [6]. The reliability of this registration method has already been demonstrated in SPECT-MRI registration [7].

Identification of homologous regions: For every voxel of the MRI volume, we determined the voxel which was anatomically homologous in the contro-lateral hemisphere. A first method consisted in defining manually the inter-hemispheric plane on MR images and using it as symmetry plane. A scaling factor was used to take into account the difference in size between the two hemispheres.

A second method used the spatial normalization scheme provided in the Statistical Parametric Mapping software package (SPM) 1 [8]. We used this method to compute non linear transformations between the MR scan of the patient and a symmetrical SPM T1 template. By using this transformation, we identified in the template the voxel corresponding to each point of the patient's MRI. The template being symmetric by construction, the homologous voxel in the contro-lateral hemisphere was obtained by symmetry. By using the inverse deformation fields, the coordinates of this homologous voxel in the patient's MRI were determined. These voxel coordinates were then transferred to the SPECT volume to define voxel-neighborboods.

Creation of an inter-hemispheric difference volume: We considered two symmetrical spherical voxel-neighborhoods (diameter 1.8 centimeters) containing 33 voxels. We calculated the means $\left(\overline{x_{1}}\right.$ and $\left.\overline{x_{2}}\right)$ and the standard deviations $\left(\sigma_{1}\right.$ and $\left.\sigma_{2}\right)$ of the voxel intensity values in both neighborhoods. Then, we calculated the difference value : $D=\frac{\overline{x_{1}-\overline{x_{2}}}}{\sqrt{\sigma_{1}^{2}+\sigma_{2}^{2}}}$. This result was stored in a volume of differences at the same coordinates as the initial voxel within the MR volume. We repeated this calculation for each MRI voxel to fill the difference volume.

\footnotetext{
${ }^{1}$ http://www.fil.ion.ucl.ac.uk/spm/
} 
Creation of a statistical volume: The statistical volume was created by testing the hypothesis $H_{0}: D=0$ against $H_{1}: D \neq 0$ at each MRI voxel and by assigning the corresponding p-value.

Voxel values of the difference volume were strongly correlated because of the limited spatial resolution of the SPECT images and the filtering process involved in the SPECT image calculation. Correlation was also introduced by the use of overlapping adjacent voxel-neighborboods. We therefore established an empirical estimation of the probability law under the null hypothesis $H_{0}$ by selecting a set of uncorrelated voxels from the difference volume. From a study of the correlation between neighbour voxels, we estimated that samples spaced by at least $18 \mathrm{~mm}$ within the brain were not correlated. Since the probability law was established under the null hypothesis, outlier voxels located in asymmetric areas should be removed. These outliers should be only few since we assumed that the asymmetry size was small with respect to the total volume of the brain. We thus removed voxels below the $1 \%$ quantile and above the $99 \%$ quantile.

From these spatially decorrelated voxels, we estimated the empirical distribution which allowed us to calculate the p-value at each voxel within the difference volume. We therefore obtained a statistical volume, to which threshold values may be applied to get a map of significant voxels corresponding to those voxels for which hypothesis $H_{0}$ was rejected.

\section{$2.2 \quad$ Validation Method}

Our aim was to determine the ability of our method to detect functional asymmetry zones of various sizes and amplitudes, in both anatomically symmetric and asymmetric brains. Simulated realistic SPECT data sets with known functional asymmetries (in size and amplitude) were used as a gold standard. Detection performances were assessed through Receiver Operating Characteristic (ROC) analysis based on measures of the overlap between known and detected asymmetries. Area under the ROC curve (AUC) was used as quality index.

Realistic SPECT simulations: To perform realistic SPECT simulation, we need to compute at the MRI resolution photon attenuation and activity maps. We used Zubal's head phantom that consists of sixty three anatomical entities manually segmented and labeled from a normal T1-weighted MRI data set 9]. The attenuation map was derived from these entities classified into seven different classes (conjunctive tissue, water, brain, bone, muscle, fat and blood). From measures performed from the SPM SPECT template, we assigned a perfusion value to each of these entities to compute the activity map [7]. These attenuation and activity maps were input parameters of the RecLBL software package created by the Lawrence Berkeley Lab [10] to simulate SPECT projections (64 projections $128 \times 128$ over $360^{\circ}$ ). Physical processes including both single photon propagation (e.g. Poisson noise and tissue attenuation) and acquisition procedures (e.g. collimator and detector response) were simulated. Tomographic reconstruction used filtered back-projection with a Hann filter (cutoff frequency : 0.4 cycle per projection bin). 
Functional asymmetries simulation: First, we simulated SPECT volumes without any anatomical asymmetry. We introduced functional asymmetric zones of various sizes and intensities in the grey matter of the temporal lobe. The asymmetric zones were spherical with a radius of 5, 10, 15 or $20 \mathrm{~mm}$. However, to model perfusion in a realistic way, only grey matter voxels were given the increased or decreased activity value (baseline activity plus or minus $10 \%, 20$ $\%, 30 \%$ or $40 \%$ ). Consequently, the volume of the asymmetric zone was 0.5 $\mathrm{cm}^{3}, 2.3 \mathrm{~cm}^{3}, 5.4 \mathrm{~cm}^{3}$ or $11 \mathrm{~cm}^{3}$, depending on the radius of the sphere. Thirty two different cases of functional asymmetries were created (combination of 4 size values and 8 amplitude values), based on an anatomically symmetric brain.

Figure 1 presents two examples of simulated SPECT volumes. The asymmetric zone extension is $11 \mathrm{~cm}^{3}$ in both figures and amplitude values are $+40 \%$ and $-40 \%$, respectively.
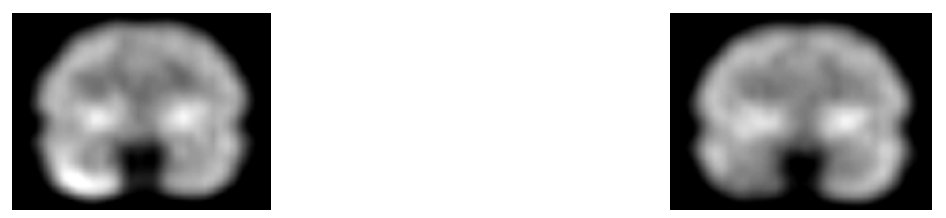

Fig. 1. Symmetric simulated SPECT with $11 \mathrm{~cm}^{3}$ asymmetric zones with amplitude values of $+40 \%$ (left) and $-40 \%$ (right) in the temporal lobe

We also assessed the ability of the method to detect asymmetric functional zones within an anatomically asymmetric brain. Therefore, we modified the spatial distribution model (based on Zubal's phantom) to introduce anatomical asymmetry. This was achieved by registering the corresponding Zubal's MRI scan to a patient's MRI scan showing obvious anatomical asymmetry of brain hemispheres. The resulting deformation fields were then applied to the spatial distribution model.

Measures of overlap: Once the asymmetry maps had been calculated (difference and statistical volumes), we calculated a degree of overlap between the actual asymmetric zone and the estimated one (at a given statistical threshold). This was achieved by counting voxels as true positives (TP), true negatives (TN), false positives (FP) and false negatives (FN). True positives are voxels belonging to both the significant zone in the statistical volume and to the actual asymmetric zone. The true negatives are voxels belonging to none of these two zones. The false positives are voxels belonging to the significant zone but not to the actual asymmetric zone. The false negatives are voxels belonging to the actual asymmetric zone but not to the significant one. Sensitivity and specificity were computed as respectively the probability to find a significant zone where there is a functional asymmetry (sensitivity $=\frac{T P}{T P+F N}$ ) and the probability not to detect significant zones where there is no functional asymmetry (specificity = $\frac{T N}{T N+F P}$ ). Receiver Operating Characteristic curves (or ROC curves) [11] were computed as plots of the true positive rate (or sensitivity) against the false pos- 


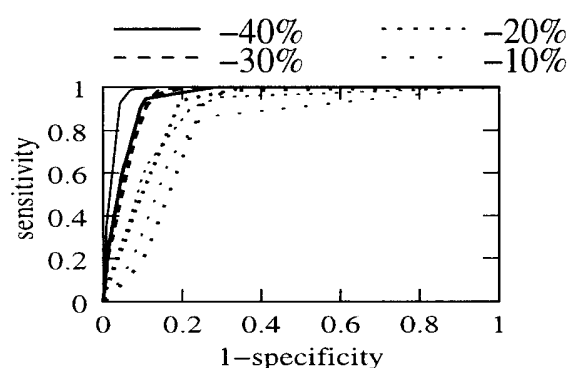

(a)

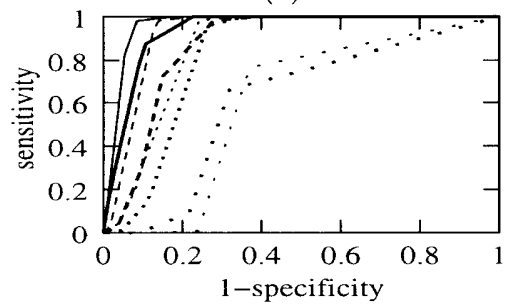

(c)

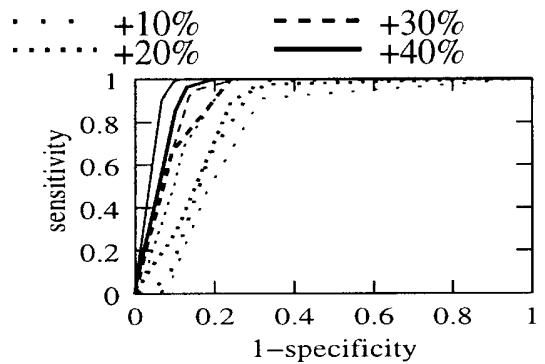

(b)

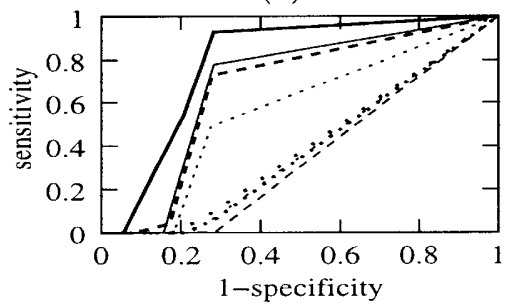

(d)

Fig. 2. ROC curves for an asymmetry of $11 \mathrm{~cm}^{3}(\mathrm{a}), 5.4 \mathrm{~cm}^{3}(\mathrm{~b}), 2.3 \mathrm{~cm}^{3}$ (c) and 0.5 $\mathrm{cm}^{3}(\mathrm{~d})$ simulated from a symmetric MRI

itive rate (1- specificity) for different statistical threshold values. Each point of ROC curves corresponds to a pair of sensitivity and specificity values deduced from a p-value threshold applied to the statistical volume. Detection efficiency was measured by the area under the ROC curve (AUC).

\section{Results}

Figure 2 shows the ROC curves obtained with the detection method applied to volumes simulated from an anatomically symmetric MRI. Symmetric voxelneighborhoods were computed using the manually defined inter-hemispheric plane. Each figure includes eight ROC curves corresponding to the eight amplitude values of the asymmetries (plus and minus 10, 20, 30, $40 \%$ of activity) for the considered extension. The AUC values are given in figure 3 as a function of the asymmetric zone amplitude and extension values. For the smallest asymmetric zone $\left(0.5 \mathrm{~cm}^{3}\right)$, the detection efficiency expressed in terms of AUC is between 0.3 and 0.6 for amplitude values of $+10,+20,-10,-20$ and $-30 \%$. It lies between 0.6 et 0.8 for the amplitude values of $+40,+30$ and $-40 \%$. It is of 0.6 for an asymmetric zone of $2.3 \mathrm{~cm}^{3}$ with a low amplitude value $(+10 \%$ and $-10 \%$ ) and close to or greater than 0.8 for all higher values of the asymmetric zone extension and amplitude.

Figure 4 shows the ROC curves obtained with the method of detection applied to volumes simulated from an anatomically asymmetric MRI. Symmetric voxel-neighborhoods were calculated by means of the spatial normalization 


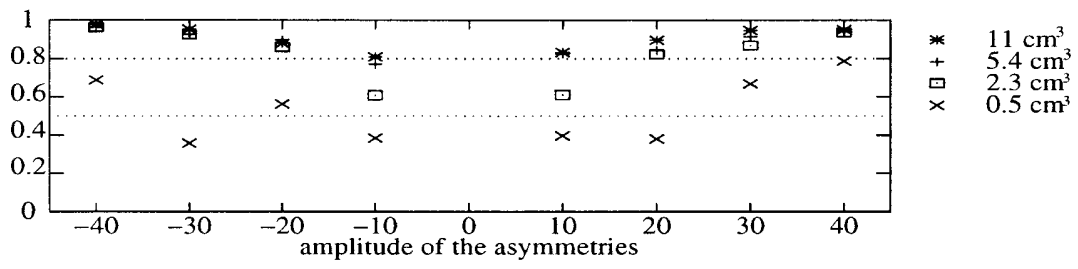

Fig. 3. AUC for asymmetries simulated from a symmetric anatomical MRI

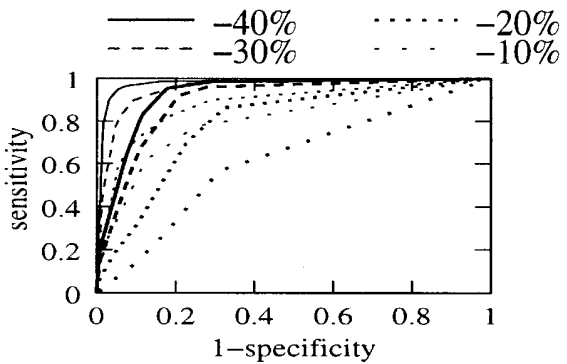

(a)

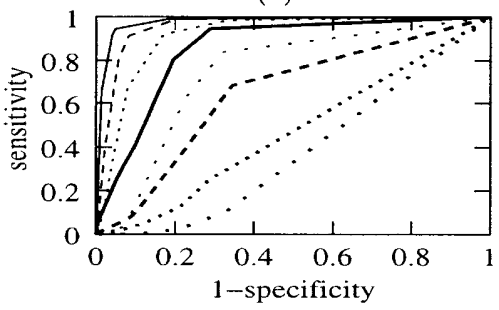

(c)

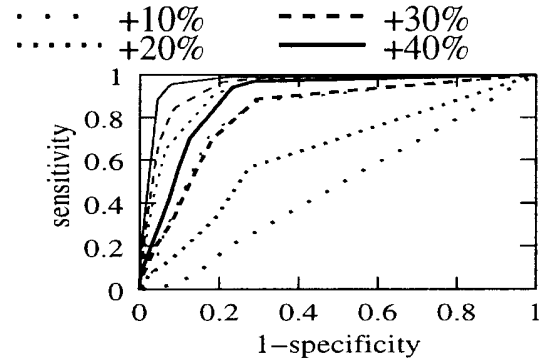

(b)

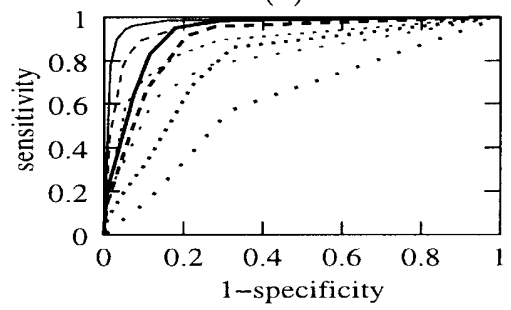

(d)

Fig. 4. ROC curves for an asymmetry of $11 \mathrm{~cm}^{3}$ (a), $5.4 \mathrm{~cm}^{3}$ (b), $2.3 \mathrm{~cm}^{3}$ (c) and 0.5 $\mathrm{cm}^{3}(\mathrm{~d})$ simulated from an asymmetric MRI

method available in the SPM package. The AUC values are given in figure 5 as a function of the asymmetric zone amplitude and extension values. The AUC values are between 0.75 and 0.98 for asymmetric zones with decreased activity, whatever the value of the asymmetric zone's extension. In the case of increased activity in a $0.5 \mathrm{~cm}^{3}$ asymmetric zone, the AUC value is around 0.5 whatever the asymmetry amplitude. For larger asymmetric zones, the AUC value is greater than 0.8 only for asymmetric zones with amplitude over $+40 \%$ in $2.3 \mathrm{~cm}^{3}$ asymmetric zones, over $+30 \%$ in $5.4 \mathrm{~cm}^{3}$ zones, and over $+20 \%$ in $11 \mathrm{~cm}^{3}$ zones.

\section{Discussion}

Our results show that, as expected, the larger and the more intense the asymmetric zone, the more efficient the detection. Within a symmetric brain, all asymmetric zones with amplitude over $20 \%$ and size over $2.3 \mathrm{~cm}^{3}$ are well detected, with AUC equal or over 0.8. These results have been obtained with the 


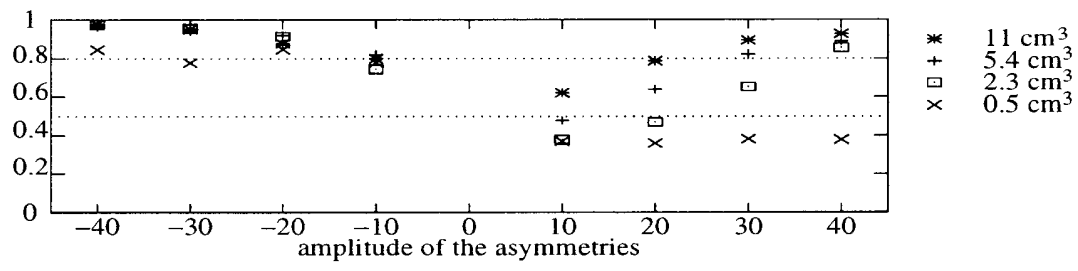

Fig. 5. AUC for asymmetries simulated from an asymmetric anatomical MRI

first way of calculating the contro-lateral homologous voxel-neighborhood (i.e. using a manual definition of the inter-hemispheric plane). The second method (based on the SPM normalization scheme) was also tested and led to slightly degraded performances (detailed results not presented here).

Within an asymmetric brain, detection performances are different depending on the sign of the asymmetry, zones with decreased uptake being better detected than zones with increased uptake. This may be related to registration errors, since a slight shift of few millimeters may change significantly the overlap degree between the actual and the detected asymmetric zone. This has to be confirmed by simulating more asymmetric zones in various locations throughout the brain.

The size of spherical voxel-neighborhood used to compute difference volumes (diameter 1.8 centimeter, 33 voxels) was a tradeoff: indeed it was large enough to take into account the spatial resolution of SPECT and to provide a sufficient number of measures, but not too large in order not to smooth and hide local differences.

An alternative to ROI or voxel-neighborhood based methods is voxel-based methods. These approaches aim at studying either inter-scan and intra-subject variability [12] or inter-scan and inter-subject variability [13], [14]. We believe that our approach studying intra-scan and intra-subject variability is different and complementary. Compared with inter-scan and inter-subject voxel-based analysis which is iffairly conservative ¿i[13], our method aims at being very sensitive and detecting most of inter-hemispheric perfusion asymmetries (with the risk to have false positive). In a second stage, our goal is to extend our detection method to determine whether this asymmetry is pathological or not, which will involve comparison with normal perfusion asymmetry measured from a control group.

\section{Conclusion}

We have presented an automatic and unsupervised method to detect functional asymmetry in SPECT images. A validation with computer-simulated data demonstrates the ability to detect asymmetric zones with relatively small extension and amplitude. Further validation based on simulated data will be performed to better characterize the performance, especially in asymmetric brain. A clinical validation will follow this first level of validation, with comparison to depth electrodes recordings and surgical outcome. 


\section{References}

1. Devous M.D., Thisted R.A., Morgan G.F. et al. SPECT brain imaging in epilepsy: a meta-analysis. J Nucl Med, 39(2):285-293, 1998.

2. Baird A.E., Donnan G.A., Austin M.C. et al. Asymmetries of cerebral perfusion in a stroke-age population. J Clin Neurosci, 6(2):113-120, 1999.

3. Kuji I., Sumiya H., Niida Y. et al. Age-related changes in the cerebral distribution of 99mTc-ECD from infancy to adulthood. J Nucl Med, 40(11):1818-1823, 1999.

4. Migneco O., Darcourt J., Benoliel J. et al. Computerized localization of brain structures in single photon emmission computed tomography using a proportional anatomical stereotaxic atlas. Comput Med Imaging Graph, 18(6):413-422, 1994.

5. Julin P., Lindqvist J., Svensson L. et al. MRI-guided SPECT measurements of medial temporal lobe blood flow in alzheimer's disease. J Nucl Med, 38(6):914919, 1997.

6. Maes F., Collignon A. et al. Multimodality image registration by maximisation of mutual information. IEEE Trans Med Imaging, 16(2):187-198, 1997.

7. Grova C., Biraben A., Scarabin J.M. et al. A methodology to validate MRI/SPECT registration methods using realistic SPECT simulated data. LNCS(MICCAI01), 2208:275-282, 2001.

8. Friston K.J., Ashburner J., Poline J.B. et al. Spatial registration and normalization of images. Hum Brain Mapp, 2:165-189, 1995.

9. Zubal I.G., Harrell C.R. et al. Computerized three-dimensional segmented human anatomy. Med Phys, 21(2):299-302, 1994.

10. Huesman R.H., Gullberg G.T., Greenberg W.L. et al. Reclbl library users manual. Technical-report pub 214, Lawrence Berkeley Lab, Univ of California.

11. Metz C.E. Roc methodology in radiologic imaging. Investigate Radiology, 21(9):720-732, 1986.

12. O'Brien T.J., O'Connor M.K., Mullan B.P. et al. Substraction ictal SPECT coregistrated to MRI in partial epilepsy: description and technical validation of the method with phantom and patient studies. Nucl Med Commun, 19(1):31-45, 1998.

13. Acton P.D. and Friston K.J. Statistical parametric mapping in functional neuroimaging: beyond PET and fMRI activation studies. Eur J Nucl Med, 25(7):663$667,1998$.

14. Lee J.D., Kim H.J., Lee B.I. et al. Evaluation of ictal brain SPET using statistical parametric mapping in temporal lobe epilepsy. Eur J Nucl Med, 27(11):1658-1665, 2000 . 